\title{
DOENÇA DO REFLUXO
} GASTROESOFÁGICO:

\section{comparação entre as formas com}

\section{e sem esofagite, em relação aos dados} demográficos e às manifestações \section{sintomáticas}

\author{
Ary NASI ${ }^{1}$, Joaquim Prado Pinto de MORAES-FILHO ${ }^{2}$, \\ Bruno ZILBERSTEIN ${ }^{3}$, Ivan CECCONELLO ${ }^{4} \mathrm{e}$ \\ Joaquim GAMA-RODRIGUES ${ }^{5}$
}

RESUMO - Racional - Os portadores da doença do refluxo gastroesofágico podem apresentar ou não esofagite por refluxo. Imagina-se que os portadores de esofagite são mais sintomáticos que os pacientes sem esofagite. Contudo, poucos estudos comparam adequadamente esses grupos de pacientes entre si. Objetivos - Comparar as formas com e sem esofagite da doença do refluxo gastroesofágico em relação à idade, sexo, presença e intensidade de sintomas. Material e Métodos - Foram estudados, por meio de entrevista clínica, 122 portadores da doença-90 com e 32 sem esofagite-identificados por estudo endoscópico do esôfago e por pHmetria esofágica prolongada. Foram considerados idade, sexo e a presença das seguintes queixas: pirose, disfagia, dor torácica não-cardíaca e sintomas respiratórios. A queixa de pirose foi analisada com mais detalhes, levando-se em conta além da presença do sintoma, a intensidade, a freqüencia e a duração da queixa. Em função dessas variáveis, a pirose foi caracterizada, quando presente, como sendo de grau leve/moderado ou intenso/muito intenso. Resultados - Não houve diferença significativa entre os grupos em relação à idade, ao sexo e à presença dos sintomas. Observou-se, contudo, em relação à intensidade da queixa de pirose, maior ocorrência de pirose caracterizada como intensa/muito intensa no grupo com esofagite. Conclusões - 1. As formas com e sem esofagite da doença do refluxo gastroesofágico são muito semelhantes em relação à idade, ao sexo e à presença dos sintomas analisados nos pacientes acometidos; 2. Apesar dos sintomas analisados estarem presentes em níveis semelhantes nos dois grupos estudados, observou-se que a ocorrência de pirose caracterizada como de maior intensidade foi mais comum no grupo de pacientes com esofagite; 3. Dentre os portadores da doença do refluxo analisados, observou-se contingente significativo de pacientes (26,2\%) que não apresentavam esofagite endoscópica; a pHmetria esofágica prolongada foi de fundamental importância para a identificação desse grupo; 4. Existe a necessidade de serem adotados conceitos mais homogêneos em relação à doença do refluxo gastroesofágico, à esofagite por refluxo e à caracterização do refluxo patológico, para obter-se maior precisão diagnóstica e para que se possa comparar, adequadamente, resultados de diferentes estudos sobre o tema.

DESCRITORES - Refluxo gastroesofágico. Esofagite péptica.

Médico Assistente Doutor da Disciplina de Cirurgia do Aparelho Digestivo da Faculdade de Medicina da Universidade de São Paulo (FMUSP). Responsável pelo Laboratório de Investigação Funcional do Esôfago.

Professor Associado. Chefe do Serviço de Esôfago da Disciplina de Gastroenterologia Clínica da FMUSP.

Professor Associado da Disciplina de Cirurgia do Aparelho Digestivo da FMUSP.

Professor Associado da Disciplina de Cirurgia do Aparelho Digestivo da FMUSP. Chefe do Serviço de Cirurgia do Esôfago do Hospital das Clínicas da FMUSP.

Professor Associado do Departamento de Gastroenterologia da FMUSP. Chefe da Disciplina de Cirurgia do Aparelho Digestivo da FMUSP.

Endereço para correspondência: Dr. Ary Nasi - Rua Oscar Freire, 1702 - apto. 88 - 05409-011 - São Paulo, SP. 


\section{INTRODUÇ̃̃O}

A doença do refluxo gastroesofágico (DRGE) é afecção de grande importância médico-social pela elevada e crescente incidência ${ }^{(2,29)} \mathrm{e}$ por determinar sintomas de intensidade variável, que se manifestam por tempo prolongado, podendo prejudicar a qualidade de vida do paciente $^{(5)}$.

Embora os dados epidemiológicos sejam escassos ${ }^{(9)}$, sabe-se que a prevalência da DRGE é grande: estima-se que $40 \%$ da população adulta apresentem, em alguma fase da vida, sintomas de refluxo gastroesofágico $(\mathrm{RGE})^{(12)}$. NEBEL et al. ${ }^{(23)}$, analisando 335 indivíduos da população geral, encontraram ocorrência diária de sintomas de refluxo em $7 \%$ e freqüência semanal ou mensal em $29 \%$ das pessoas entrevistadas.

Com o desenvolvimento dos dispositivos de monitorização prolongada do $\mathrm{pH}$ intraluminar esofágico, ampliou-se o conhecimento fisiopatológico da doença, chegando-se, assim, à concepção atual da DRGE, onde entende-se que o RGE é o principal fator agressivo responsável pelo aparecimento da doença, que pode determinar ou não esofagite de refluxo (ER), na dependência de outros fatores ainda pouco conhecidos.

Do desequilíbrio entre os fatores agressivos da mucosa esofágica, fundamentalmente o RGE e os fatores defensivos, podem surgir alterações estruturais da parede esofágica, identificáveis de forma macroscópica pelo exame endoscópico e/ou, no plano microscópico, pela análise histopatológica de material de biopsia obtido na região, caracterizando o que se denomina de ER.

É importante comentar, no entanto, que pode não haver o aparecimento de esofagite, mesmo ocorrendo RGE em níveis considerados patológicos, desde que os mecanismos de defesa da mucosa sejam suficientemente ativos para evitar alterações estruturais da mesma. Caracteriza-se, dessa forma, um grupo de pacientes que apresentam o que se denomina de RGE sem esofagite.

Convém salientar que os pacientes com DRGE sem esofagite são, especialmente em nosso meio, pouco diagnosticados pela pequena disponibilidade assistencial da pHmetria esofágica prolongada e, também, pelo desconhecimento dessa forma de manifestação clínica por muitos médicos. As repercussões negativas desse fato, do ponto de vista médico-assistencial, são evidentes.

No presente trabalho, considerou-se justificável conceituar a DRGE como a afecção representada por manifestações sintomáticas e/ou alterações anatômicas esofágicas (macro ou microscópicas) decorrentes do RGE e, portanto, incluir entre os portadores da doença os pacientes que apresentem:

- ER ao estudo endoscópico, independentemente da presença de sintomas e da comprovação do refluxo;

- RGE em níveis patológicos à pHmetria esofágica, independentemente da presença de sintomas;
- sintomas de RGE (típicos ou atípicos) que tenham refluxo documentado como causa das queixas clínicas, independentemente de o refluxo ser ou não classificado como patológico.

O objetivo deste estudo foi analisar pacientes com DRGE nas formas com e sem esofagite, comparando-os entre si em relação aos dados demográficos e sintomas apresentados.

\section{CASUÍSTICA E MÉTODOS}

Foram analisados retrospectivamente 565 prontuários referentes a pacientes submetidos a pHmetria esofágica. Destes, foram selecionados 122 que apresentavam elementos suficientes para análise no presente estudo e que preenchiam totalmente os critérios de inclusão estabelecidos.

Caracterizaram-se os pacientes como portadores de DRGE quando os mesmos apresentavam ER ou refluxo patológico ou sintomático sem esofagite. $\mathrm{O}$ critério para essas conceituações baseou-se na avaliação endoscópica e de pHmetria esofágica, conforme exposto a seguir:

- considerou-se ER quando o estudo endoscópico detectava tal alteração na forma não-erosiva, erosiva, ou complicada, independentemente do resultado da pHmetria esofágica;

- considerou-se refluxo patológico ou fisiológico sintomático sem esofagite quando o estudo endoscópico não detectava alterações sugestivas de esofagite, mas a pHmetria esofágica identificava RGE em níveis patológicos (refluxo patológico), ou episódios de refluxo associados à ocorrência de sintomas durante a monitorização com refluxo em níveis fisiológicos (refluxo fisiológico sintomático).

Os grupos estudados a partir de tal conceituação serão designados simplesmente como portadores da DRGE nas formas com e sem esofagite.

Foram considerados critérios de inclusão:

1. presença no prontuário de anamnese detalhada, incluindo observação minuciosa sobre as características clínicas de pirose, disfagia, dor torácica e sintomas respiratórios;

2. diagnóstico confirmado de DRGE, baseado no resultado do exame endoscópico e/ou pHmétrico;

3. presença de avaliação endoscópica descritiva detalhada do esôfago, estômago e duodeno;

4. realização de $p H$ metria esofágica prolongada em intervalo de tempo igual ou inferior a 15 dias, em relação à avaliação endoscópica;

5. não utilização de qualquer tratamento farmacológico que pudesse interferir com o grau de refluxo e de esofagite, no período compreendido entre a execução da endoscopia e da pHmetria (alcalinos, prócineticos, bloquedores $\mathrm{H} 2$, inibidores da bomba protônica, drogas relaxadoras de musculatura lisa e drogas com potencial reconhecido de agressão à mucosa esofágica). 
Nasi A, Moraes-Filho JPP de, Zilberstein B, Cecconello I, Gama-Rodrigues J. Doença do refluxo gastroesofágico: comparação entre as formas com e sem esofagite, em relação aos dados demográficos e às manifestações sintomáticas

A entrevista clínica e os exames de manometria e pHmetria foram realizados sempre pelo mesmo examinador.

\section{ENTREVISTA CLÍNICA}

Além da caracterização dos pacientes quanto ao sexo e à idade, pesquisou-se a presença dos seguintes sintomas: pirose, disfagia, dor torácica não-cardíaca (DTNC) e manifestações respiratórias potencialmente atribuíveis ao refluxo. Quando existia a presença da queixa de pirose, detalhava-se um pouco mais o interrogatório clínico, objetivando-se avaliar o tempo de existência do sintoma, a intensidade e freqüência do mesmo. Com base nesses parâmetros, a queixa de pirose era classificada em leve/moderada ou intensa/muito intensa.

\section{ANÁLISE ESTATÍSTICA}

Para a análise dos resultados foram utilizados testes não-paramétricos, levando-se em consideração a natureza das variáveis estudadas. Foram aplicados os seguintes testes:

- Teste de Mann-Whitney para duas amostras independentes $^{(28)}$, com a finalidade de comparar os dois grupos estudados em relação à idade. Considerando-se o tamanho das amostras, este teste foi aplicado com aproximação à curva normal (z calculado).

- Teste do qui-quadrado para tabelas de freqüência ${ }^{(28)}$, com o objetivo de comparar os dois grupos em relação ao sexo e aos sintomas apresentados. Em alguns casos, tendo em vista as restrições de Cochran, aplicou-se o teste exato de Fisher ${ }^{(28)}$.

Em todos os testes fixou-se em 0,05 ou $5 \%$ (alfa menor ou igual a $0,05)$ o nível para rejeição da hipótese de nulidade, assinalando-se com um asterisco os valores significativos.

\section{RESULTADOS}

Dos 122 pacientes analisados, $90(73,8 \%)$ apresentavam a forma esofagite de refluxo (com ER) e 32 (26,2\%), a forma refluxo patológico ou fisiológico sintomático sem esofagite (sem ER) da DRGE.
A idade variou de 16 a 78 anos (média de 42,6 anos) no grupo com ER, e de 20 a 74 anos (média de 40,7 anos) no grupo sem ER. Não houve diferença significativa quanto à idade dos pacientes nos dois grupos $(P=0,4602)$

Em relação ao sexo, observou-se que $56(62,2 \%)$ pacientes eram do sexo masculino e 34 (37,8\%) eram do sexo feminino no grupo com ER, e que $14(43,7 \%)$ pacientes eram do sexo masculino e 18 (56,3\%) eram do sexo feminino no grupo sem ER. Não houve diferença significativa quanto à distribuição entre os sexos nos dois grupos $(P<0,10)$. São apresentados na Tabela 1, os dados demográficos dos pacientes estudados.

\section{SINTOMAS}

\section{Pirose}

A ocorrência de pirose nas formas com e sem ER foi de 97,8\% e $90,6 \%$, respectivamente, não havendo diferença significativa entre tais níveis de ocorrência (teste exato de Fisher, $P=0,1124$ ).

Quanto à caracterização da pirose, observou-se que a ocorrência de pirose intensa ou muito intensa foi significativamente maior $(P<0,01)$ na forma com ER -33 pacientes $(37,5 \%)$, do que na sem ER -3 pacientes $(10,3 \%)$

\section{Disfagia}

Dezessete $(18,9 \%)$ pacientes do grupo com ER e dois $(6,2 \%)$ do grupo sem ER apresentavam queixa de disfagia à entrevista clínica. A análise estatística não demonstrou diferença significativa entre a ocorrência de disfagia nas duas formas de apresentação da doença (teste exato de Fisher, $P=0,0731$ ).

\section{Dor torácica não-cardíaca}

Quatro (4,4\%) pacientes do grupo com ER e cinco (15,6\%) do grupo sem ER apresentavam queixa de dor torácica. Não houve diferença significativa entre tais valores (teste exato de Fisher, $P=0,0521$ ).

TABELA 1 - Distribuição dos pacientes quanto à presença de esofagite, ao sexo e à idade

\begin{tabular}{|c|c|c|c|c|c|c|}
\hline & \multicolumn{2}{|c|}{ Com ER } & \multicolumn{2}{|c|}{ Sem ER } & \multicolumn{2}{|c|}{ TOTAL } \\
\hline & $\mathbf{N}$ & $\%$ & $\mathbf{N}$ & $\%$ & $\mathbf{N}$ & $\%$ \\
\hline Número de pacientes & 90 & 73,8 & 32 & 26,2 & 122 & 100,0 \\
\hline Sexo masculino & 56 & 62,2 & 14 & 43,7 & 70 & 57,4 \\
\hline Sexo feminino & 34 & 37,8 & 18 & 56,3 & 52 & 42,6 \\
\hline Idade (anos): min-max (média) & \multicolumn{2}{|c|}{$16-78(42,6)$} & \multicolumn{2}{|c|}{$20-74(40,7)$} & \multicolumn{2}{|c|}{$16-78(42,1)$} \\
\hline TOTAL & 90 & 100,0 & 32 & 100,0 & 122 & 100,0 \\
\hline
\end{tabular}

$\mathrm{ER}=$ esofagite de refluxo 
Nasi A, Moraes-Filho JPP de, Zilberstein B, Cecconello I, Gama-Rodrigues J. Doença do refluxo gastroesofágico: comparação entre as formas com e sem esofagite, em relação aos dados demográficos e às manifestações sintomáticas

\section{Sintomas respiratórios}

Quatro $(4,4 \%)$ pacientes do grupo com ER e dois $(6,2 \%)$ do grupo sem ER referiam presença de sintomas respiratórios à entrevista clínica. A análise estatística não demonstrou diferença significativa entre tais valores (teste exato de Fisher, $P=0,4980$ ).

Apresenta-se na Tabela 2 a presença dos sintomas analisados nos dois grupos.

\section{DISCUSSÃO}

Constatou-se que $26,2 \%$ dos pacientes caracterizados como portadores da DRGE não apresentam esofagite identificável pelo estudo endoscópico. A confrontação desse número com os resultados de outros autores é dificultada pela grande variedade de critérios de definição adotados.

KASAPIDIS et al. ${ }^{(13)}$, estudando 42 pacientes com sintomas sugestivos de DRGE e RGE patológico comprovado por pHmetria, constataram que 21 pacientes $(50,0 \%)$ apresentavam ER e que os 21 restantes, apesar de terem RGE patológico, não apresentavam alterações da mucosa esofágica detectáveis pela endoscopia. Os dados obtidos nesse estudo não podem ser diretamente comparados com os do presente trabalho pois, de maneira diferente do critério adotado na presente casuística, na pesquisa daqueles autores consideraram-se como portadores de DRGE somente os pacientes com sintomas sugestivos da afecção que tivessem RGE patológico comprovado pela pHmetria. O critério adotado por eles não nos parece adequado, por desconsiderar contingente significativo de pacientes sintomáticos que apresentam esofagite e não têm RGE patológico à pHmetria.

MASCLEE et al. ${ }^{(18)}$, em 67 pacientes com sintomas sugestivos de DRGE estudados por meio de endoscopia digestiva alta e pHmetria esofágica prolongada, observaram que $44(65,7 \%)$ apresentavam esofagite e 23 (34\%), não. Esse estudo aproxima-se mais do presente trabalho, em termos da distribuição das formas com e sem ER da DRGE. Entretanto, constata-se que nove pacientes $(13,4 \%$ do total analisado) apresentavam estudos endoscópico e pHmétrico normais, tornando questionável o diagnóstico da DRGE nessa situação, apesar da presença de sintomas sugestivos da doença.
Os resultados do presente trabalho, consubstanciados pelos demais apresentados, indicam que contingente significativo dos pacientes com DRGE não apresenta ER identificável ao estudo endoscópico, sendo necessária a realização de pHmetria esofágica para caracterização desse grupo. Depreende-se, ainda, que a grande diversidade de conceituações utilizadas de DRGE dificulta comparações de resultados.

Analisando-se eventuais diferenças entre os grupos com e sem ER em termos de idade e sexo, observou-se que, apesar de a média de idade ser superior na forma com ER (45,3 anos) em relação à observada na forma sem ER (40,7 anos), não se constatou significância estatística nessa diferença $(P=0,4602)$

Quanto ao sexo, observou-se leve predomínio do sexo masculino no grupo com ER $(62,2 \%)$ e do sexo feminino no grupo sem ER $(56,3 \%)$. Tendo em vista, contudo, o tamanho das amostras avaliadas, não foi possível rejeitar a hipótese de igualdade de distribuição quanto ao sexo entre os grupos $(P<0,10)$.

MASCLEE et al. ${ }^{(18)}$ encontraram resultados semelhantes em relação à distribuição quanto ao sexo e à idade nos grupos com e sem esofagite. Assim como na presente série, entretanto, quase foram atingidos níveis de significância estatística quanto ao sexo $(P=0,07)$.

KASAPIDIS et al. ${ }^{(13)}$, analisando as formas com e sem esofagite da DRGE, observaram igualdade de distribuição quanto à idade, mas não fizeram referências em relação ao sexo.

Várias publicações ${ }^{(3,10,21,29,32)}$ descrevem que a prevalência da DRGE em adultos aumenta com a idade, atingindo níveis mais elevados após os 40 anos. Tais fatos foram consubstanciados no presente estudo ao verificar-se que a média de idade dos pacientes estudados era acima dos 40 anos.

A história clínica é de grande importância para o diagnóstico da DRGE, sendo a pirose o sintoma mais característico da afecção ${ }^{(8,31)}$, apesar de também ocorrer em outras doenças do trato digestivo alto, como na úlcera péptica ${ }^{(22,26)}$ e nas gastrites ${ }^{(20)}$.

A correlação entre o sintoma pirose e o processo inflamatório esofágico foi proposta inicialmente por WINKELSTEIN ${ }^{(33)}$, ao considerar que a pirose seria decorrente da ação da secreção gástrica em contato com a mucosa esofágica lesada, empregou o termo esofagite péptica para descrever tal inflamação. Mais tarde, ALLISON(1) usou,

TABELA 2 - Distribuição dos pacientes quanto à presença dos sintomas analisados

\begin{tabular}{|c|c|c|c|c|c|c|}
\hline \multirow[t]{2}{*}{ SINTOMAS } & \multicolumn{2}{|c|}{ Com ER } & \multicolumn{2}{|c|}{ Sem ER } & \multicolumn{2}{|c|}{ TOTAL } \\
\hline & $\overline{\mathbf{N}}$ & $\%$ & $\mathbf{N}$ & $\%$ & $\mathbf{N}$ & $\%$ \\
\hline Presença de pirose $(P=0,1124)$ & 88 & 97,8 & 29 & 90,6 & 117 & 95,9 \\
\hline Presença de disfagia $(P=0,0731)$ & 17 & 18,9 & 2 & 6,2 & 6 & 15,6 \\
\hline Presença de DTNC $\quad(P=0,0521)$ & 4 & 4,4 & 5 & 15,6 & 9 & 7,4 \\
\hline Presença de sintomas respiratórios $(P=0,4980)$ & 4 & 4,4 & 2 & 6,2 & 6 & 15,6 \\
\hline
\end{tabular}

$\mathrm{ER}=$ esofagite de refluxo; DTNC $=$ dor torácica não-cardíaca $P<0,05^{*}$ 
Nasi A, Moraes-Filho JPP de, Zilberstein B, Cecconello I, Gama-Rodrigues J. Doença do refluxo gastroesofágico: comparação entre as formas com e sem esofagite, em relação aos dados demográficos e às manifestações sintomáticas

pela primeira vez, o termo ER, deixando mais clara a participação do RGE na fisiopatologia da enfermidade.

Dos 122 pacientes avaliados no presente estudo, $117(95,9 \%)$ apresentavam sintoma de pirose, $88(97,8 \%)$ no grupo com e 29 $(90,6 \%)$ no grupo sem ER, não havendo diferença estatisticamente significativa entre os grupos. Quando se considerou a pontuação utilizada para caracterizar o sintoma quanto à intensidade, freqüência e duração, observou-se, entretanto, que ao ser empregado parâmetro de observação mais detalhado, os pacientes com ER apresentaram maior ocorrência do sintoma caracterizado como intenso ou muito intenso $(37,5 \%)$ do que os pacientes sem ER $(10,3 \%)$.

Essa consideração merece destaque porque permite, a grosso modo, afirmar que quanto mais intensa é a pirose apresentada pelo paciente, maior é a probabilidade de que exista ER. Essa observação não é nova. De fato, KLAUSER et al. ${ }^{(14)}$, analisando a presença de pirose em pacientes com DRGE e RGE patológico evidenciado pela pHmetria esofágica, observaram maior ocorrência do sintoma no grupo com esofagite em relação ao sem esofagite $(84 \%$ X $60 \%$, respectivamente; $P=0,013$ ). Diferentemente do presente trabalho, contudo, somente os pacientes que apresentavam erosões da mucosa esofágica ao estudo endoscópico foram caracterizados como portadores de esofagite.

Os resultados obtidos no presente trabalho indicam que a incidência do sintoma pirose, sem considerar sua intensidade, é semelhante nas formas com e sem ER da DRGE; portanto, na investigação clínica de pacientes com suspeita da doença, não se pode inferir sobre a presença ou não de ER pela simples existência da queixa de pirose. Como já se referiu, todavia, ao se utilizar sistema de pontuação mais detalhado, constata-se que, na presença de pirose classificada como intensa ou muito intensa, a probabilidade de existência de ER ao estudo endoscópico é maior.

Como se sabe, outros sintomas além da pirose, como regurgitação, sialorréia, odinofagia, disfagia, DTNC e manifestações respiratórias, também podem ser determinados pela DRGE.

Dos 122 pacientes analisados, observou-se presença do sintoma disfagia em 19 (15,6\%), sendo 17 (18,9\%) no grupo com e dois $(6,2 \%)$ no grupo sem ER. Apesar de os resultados demonstrarem maior ocorrência de disfagia no grupo com ER, não foi possível, do ponto de vista estatístico, rejeitar a hipótese de igualdade de incidência da queixa entre os grupos estudados $(P=0,0731)$. Destaca-se o fato de $6,2 \%$ dos pacientes sem esofagite também apresentarem o sintoma, reforçando a ocorrência e a importância das alterações motoras esofágicas na DRGE.

A ocorrência de disfagia é freqüentemente associada à presença de complicações da esofagite de refluxo, quando existe redução do calibre intraluminar. Não obstante, o sintoma também pode ser observado em pacientes com esofagite não-complicada e mesmo em portadores de RGE sem esofagite, como observamos no presente trabalho.

A dor torácica constitui motivo de grande preocupação para médicos e pacientes, por levantar a suspeita de isquemia miocárdica, entidade que apresenta elevados índices de mortalidade.

Do ponto de vista fisiopatológico, o envolvimento esofágico na gênese da DTNC depende, primordialmente, do RGE e de distúrbios motores do esôfago. Com efeito, as primeiras publicações relacionando DTNC com RGE foram de DeMEESTER et al. ${ }^{(7)}$ que, investigando 50 pacientes com DTNC, encontraram RGE patológico evidenciado pela pHmetria esofágica em 23 (46\%). Estudos subseqüentes ${ }^{(11,24,27)}$ observaram que entre $9 \%$ e $33 \%$ dos pacientes com DTNC submetidos ao exame pHmétrico apresentavam o sintoma associado à ocorrência de RGE durante a monitorização.

No presente estudo observou-se que dos 122 pacientes estudados, nove $(7,4 \%)$ apresentavam DTNC, quatro $(4,4 \%)$ no grupo com e cinco $(15,6 \%)$ no grupo sem ER, documentando o fato de existir associação de DTNC com DRGE nas formas com e sem ER da doença. Apesar de a ocorrência do sintoma ter sido maior na forma sem ER, não foi possível rejeitar a hipótese de igualdade de ocorrência de DTNC nos dois grupos estudados $(P=0,0521)$.

KUSTER et al. ${ }^{(15)}$, analisando 109 pacientes com sintomas sugestivos de DRGE, constataram que 21 (19\%) apresentavam DTNC. Apesar de não compararem a ocorrência do sintoma nas formas com e sem esofagite da afecção, notaram não haver relação entre a intensidade do sintoma e a gravidade da esofagite.

Existem diversas publicações associando DTNC com DRGE; entretanto, em nenhuma delas se observa a análise da ocorrência do sintoma nas formas com e sem ER da doença, conforme apresentado no presente trabalho.

A ocorrência de manifestações respiratórias, como tosse, asma e pneumonias recorrentes, também pode ser notada em pacientes com DRGE, especialmente em crianças e em idosos. Duas teorias têm sido aventadas para explicar a relação entre RGE e sintomas respiratórios. A mais recente sugere mecanismo reflexo esôfago-brônquico, no qual o RGE estimularia constrição brônquica determinando sintomas $^{(16,17)}$. Outra teoria baseia-se na ocorrência de microaspirações do RGE para as vias respiratórias, desencadeando manifestações clínicas ${ }^{(4,19)}$

No presente estudo observaram-se que seis $(4,9 \%)$ pacientes apresentavam sintomas respiratórios, quatro $(4,4 \%)$ no grupo com e dois $(6,2 \%)$ no grupo sem ER. Não houve diferença significativa entre os grupos $(P=0,4980)$, atestando a associação de sintomas respiratórios com DRGE independentemente da presença de esofagite.

KUSTER et al. ${ }^{(15)}$, estudando 109 pacientes com sintomas sugestivos de DRGE, constataram ocorrência de manifestações respiratórias em 37 (34\%). Apesar de não analisarem a ocorrência de 
Nasi A, Moraes-Filho JPP de, Zilberstein B, Cecconello I, Gama-Rodrigues J. Doença do refluxo gastroesofágico: comparação entre as formas com e sem esofagite, em relação aos dados demográficos e às manifestações sintomáticas

tais sintomas em relação à presença ou não de esofagite, notaram não haver relação entre a intensidade da manifestação respiratória e a gravidade da esofagite.

\section{CONCLUSÕES}

1. Dentre os portadores da DRGE existe um contingente expressivo de pacientes $(26,2 \%)$ que não apresenta esofagite identificável pelo estudo endoscópico. A realização de pHmetria esofágica prolongada é fundamental para a identificação desse grupo.

2. A distribuição quanto ao sexo e à idade dos pacientes foi semelhante $(P<0,10$ e 0,4602 , respectivamente) nos grupos com e sem esofagite.

3. A ocorrência de sintomas respiratórios, pirose, disfagia e DTNC foi semelhante nos grupos com e sem esofagite. Foi observada, entretanto, diferença significativa na caracterização do tipo de pirose apresentado, predominando pirose intensa ou muito intensa nos pacientes com esofagite $(P<0,01)$
4. Há polêmica em relação a alguns critérios conceituais importantes (conceito de ER, de refluxo patológico e da própria DRGE). É necessário que se aprimorem tais critérios conceituais para maior precisão diagnóstica e para comparar resultados de diferentes estudos sobre o tema.

5. A nosso ver, em relação ao critério conceitual da DRGE, já existem subsídios para conceituar-se a doença de maneira mais abrangente, como foi efetuado no presente estudo, "como sendo a afecção representada por manifestações sintomáticas e/ou alterações anatômicas esofágicas (macro ou microscópicas) decorrentes do RGE". Portanto, deve-se incluir entre os portadores da doença os pacientes que apresentem:

- ER ao estudo endoscópico, independentemente da presença de sintomas e da comprovação do refluxo;

- RGE em níveis patológicos à pHmetria esofágica, independentemente da presença de sintomas;

- sintomas sugestivos de RGE que tenham refluxo documentado como causa das queixas clínicas, independentemente de o refluxo ser ou não classificado como patológico.

Nasi A, Moraes-Filho JPP de, Zilberstein B, Cecconello I, Gama-Rodrigues J. Gastroesophageal reflux disease: comparison between patients with and without esophagitis, concerning age, gender and symptoms. Arq Gastroenterol 2001;38(3):109-115.

ABSTRACT-Background-Patients with gastroesophageal reflux disease may or may not have endoscopic esophagitis; there are few studies comparing these groups among themselves. Objectives - This study was designed in order to evaluate differences between patients with gastroesophageal reflux disease with and without esophagitis. Patients/Methods - A hundred and twenty-two patients with gastroesophageal reflux disease characterized by esophageal endoscopy and pHmetry were included, 90 with and 32 without esophagitis. Assessment involved an anamnesis, including the following data: age, sex, heartburn, dysphasia, non-cardiac chest pain and respiratory symptoms. Heartburn was analyzed in more detail, its duration, intensity and periodicity being determined. Results - No statistical significant difference was observed between the groups, regarding age, sex or presence of symptoms. However, in the group with esophagitis, heartburn classified as severe or very severe was more frequent. Conclusions - 1. The groups of patients with or without esophagitis analyzed were very similar concerning age, gender and presence of symptoms. However, regarding the heartburn's intensity, it was more intense in the group with esophagitis. 2. Among patients with gastroesophageal reflux disease, there is a large number of cases without esophagitis (26.2\%) and that prolonged pH-monitoring is fundamental in its identification; 3. A better definition of reflux disease, esophagitis and pathological reflux is needed, in order to allow better diagnostic accuracy and comparisons in different studies on this subject.

HEADINGS - Gastroesophageal reflux. Esophagitis, peptic.

\section{REFERÊNCIAS BIBLIOGRÁFICAS}

1. Allison PR. Reflux oesophagitis, sliding hiatal hernia, and the anatomy of repair Surg Gynecol Obstet 1951;92:419-31.

2. Bettarello A, Penteado JF, Meneghelli U, Dani R, Fernandes RST, Sugisawa S, Lima JP, Castro LP. A importância do refluxo gastroesofágico e da esofagite de refluxo na clínica diária. Folha Médica 1987;95:66-71.

3. Brunnen PL, Karmody AM, Needham CD. Severe peptic oesophagitis. Gut 1969;10:831-7.

4. Chernow B, Johnson LF, Janowitz WR, Castell DO. Pulmonary aspiration as a consequence of gastro-oesophageal reflux: a diagnostic approach. Dig Dis Sci 1979;24:839-44

5. Coley CM, Barry MJ, Spechler SJ. Initial medical v. surgical therapy for complicated or chronic gastroesophageal reflux disease (GERD). A cost effectiveness analysis. Gastroenterology 1993;104:A5.
6. De Dombal FT, Horrcks JC, McAdam WA, Morgan AG, Pacsoo C, Darnborough A. Computer-aided diagnosis of dyspepsia: a prospective series with emphasis upon comparison with endoscopic findings. Gut 1975;16:842.

7. DeMeester TR, O'Sullivan GC, Bermudez G, Midell AL, Cimochowski GE, O'Drobinak J. Esophageal function in patients with angina-type chest pain and normal coronary arteriograms. Ann Surg 1982;196:488-97.

8. Fisher RS, Malmud LS, Roberts GS, Lobis IF. Gastroesophageal scintiscanning to detect and quantitative GE reflux. Gastroenterology 1976;70:301-8.

9. Fuchs KH, DeMeester TR, Albertucci M. Specificity and sensitivity of objective diagnosis of gastroesophageal reflux disease. Surgery 1987;102:575-9.

10. Heading RC. Epidemiology of oesophageal reflux disease. Scand J Gastroenterol $1989 ; 24: 72-6$

11. Janssens J, Vantrappen G, Ghillebert G. 24-hour recording of esophageal pressure and $\mathrm{pH}$ in patients with noncardiac chest pain. Gastroenterology 1986;90:1978-84

12. Jones PT. General practice in London. Br J Gen Pract 1990;40(340):480. 
Nasi A, Moraes-Filho JPP de, Zilberstein B, Cecconello I, Gama-Rodrigues J. Doença do refluxo gastroesofágico: comparação entre as formas com e sem esofagite, em relação aos dados demográficos e às manifestações sintomáticas

13. Kasapidis P, Xynos E, Mantides A, Chrysos E, Memonakou M, Nikolopoulos N, Vassilakis JS. Differences in manometry and 24-h ambulatory pH-metry between patients with and without endoscopic or histological esophagitis in gastroesophageal reflux disease. Am J Gastroenterol 1993;88:1893-9.

14. Klauser AG, Schindlbeck NE, Muller-Lissner SA. Symptoms in gastrooesophageal reflux disease. Lancet 1990;335:205-8.

15. Kuster E, Ros E, Toledo-Pimentel V, Pujol A, Bordas JM, Grande L, Pera C. Predictive factors of the long term outcome in gastroesophageal reflux disease: six year follow up of 107 patients. Gut 1994;35:8-14.

16. Mansfield LE, Hameister HH, Spaulding HS, Smith NJ, Glab N. The role of the vague nerve in airway narrowing caused by intraesophageal hydrochloric acid provocation and esophageal distention. Ann Allergy 1981;47:431-4.

17. Mansfield LE, Stein MR. Gastroesophageal reflux and asthma: a possible reflux mechanism. Ann Allergy 1978;41:224-6.

18. Masclee AA, De Best AC, De Graaf R, Cluysenaer OJ, Jansen JB. Ambulatory 24 hour $\mathrm{pH}$-metry in the diagnosis of gastroesophageal reflux disease. Determination of criteria and relation to endoscopy. Scand J Gastroenterol 1990;25:225-30.

19. Mays EE, Dubois JJ, Hamilton GB. Pulmonary fibrosis associated with tracheobronchial aspiration. Chest 1976;69:512-5.

20. Millat B, Pignon JP. When must we consider reflux esophagitis? In: Giuli R, McCallum RW, editors. Benign lesions of the esophagus and cancer. Berlin: Springer-Verlag; 1989. p.99-107.

21. Mold JW, Reed LE, Davis AR, Allen ML, Decktor DL, Robinson M. Prevalence of gastroesophageal reflux in elderly patients in a primary care setting. Am J Gastroenterol 1991;86:965-70.

22. Moraes-Filho JPP. Contribuição ao estudo da esofagite de refluxo em portadores de úlcera duodenal [tese de doutorado]. São Paulo: Faculdade de Medicina da Universidade de São Paulo; 1971.
23. Nebel OT, Fornes MF, Castell DO. Symptomatic gastro-oesophageal reflux: incidence and precipiting factors. Dig Dis Sci 1976;21:953-6.

24. Peters L, Maas L, Petty D, Dalton C, Penner D, Wu W, Castell D, Richter J. Spontaneous noncardiac chest pain: evaluation by 24 -hour ambulatory esophageal motility and $\mathrm{pH}$ monitoring. Gastroenterology 1988;94:878-86.

25. Pope II CE. Chest pain: heart?, gullet?, both?, neither?. JAMA 1982;248:2315

26. Schiller LR. Epidemiology, clinical manifestations and diagnosis. In: Wyngaarden JB, Smith LH, Bennett JC. editors, Cecil textbook of medicine. Philadelphia: W.B. Saunders; 1992. v.1, p.656-8

27. Schofield PM, Whorwell PJ, Brooks NH, Bennett DH, Jones PE. Oesophagea function in patient with angina pectoris: a comparasion of patients with norma coronary angiogram and patients with coronary artery disease. Digestion 1989;42:70-8.

28. Siegel S. Estadística no paramétrica. México: Trilhas; 1975

29. Stoker DL, Williams JG, Leicester RG, Colin-Jones DG. Oesophagitis - a five year review. Gut 1988;29:1450.

30. Tuttle SG, Bettarello A, Grossman MI. Gastroesophageal regurgitation. JAMA 1960;176:498-500.

31. Tuttle SG, Grossman MI. Detection of gastro-oesophageal reflux by simultaneous measurement of intraluminal pressure and $\mathrm{pH}$. Proc Soc Exp Biol Med. 1958;98:225.

32. Wienbeck M, Barnert J. Epidemiology of reflux disease and reflux esophagitis Scand J Gastroenterol 1989;24:7-13.

33. Winkelstein A. Peptic esophagitis. A new clinical entity. JAMA 1935;104:906-8. 